\title{
Overexpression of TRIB3 promotes angiogenesis in human gastric cancer
}

\author{
SHAOTING DONG ${ }^{1,4}$, JIANLING XIA ${ }^{1}$, HONGQIANG WANG ${ }^{1,5}$, LI SUN $^{1}$, ZHENZHEN WU $^{1}$, \\ JIANPING BIN ${ }^{2}$, YULIN LIAO ${ }^{2}$, NAILIN $^{3}$ and WANGJUN LIAO ${ }^{1}$
}

\begin{abstract}
Departments of ${ }^{1}$ Oncology and ${ }^{2}$ Cardiology, Nanfang Hospital, Southern Medical University, Guangzhou, Guangdong 510515, P.R. China; ${ }^{3}$ Karolinska Institutet, Department of Medicine-Solna, Clinical Pharmacology Group, Karolinska University Hospital-Solna, Stockholm 17176, Sweden; ${ }^{4}$ Department of Oncology, Huizhou Municipal Central Hospital, Huizhou, Guangdong 516002; ${ }^{5}$ Department of Oncology, Zhoushan Hospital, Zhoushan, Zhejiang 316000, P.R. China
\end{abstract}

Received January 28, 2016; Accepted March 8, 2016

DOI: 10.3892/or.2016.5017

\begin{abstract}
Tribbles homolog 3 (TRIB3) plays important roles in many types of malignancies. However, whether TRIB3 is involved in the development or progression of gastric cancer (GC) remains unclear. In this study, we analyzed TRIB3 expression in GC tissues from 191 GC patients categorized with stage I to IV disease, to examine the role of TRIB3 in GC, and examined the relationship between TRIB3 and tumor angiogenesis. We found that TRIB3 expression was significantly higher in GC tissues than that in adjacent non-tumor tissues. TRIB3 expression was associated with VEGF-A and tumor microvessel density, as well as overall TNM stage, T stage, $\mathrm{N}$ stage, and distant metastasis in GC tissues. Furthermore, TRIB3 silencing downregulated VEGF-A expression in GC cells, which subsequently suppressed endothelial cell recruitment and vessel formation. In conclusion, overexpression of TRIB3 is associated with tumor angiogenesis and a poor prognosis in patients with GC. Our findings indicate that TRIB3 is a promising target for anti-angiogenic therapy in GC.
\end{abstract}

\section{Introduction}

Gastric cancer (GC) is one of the most common malignant tumors, and is responsible for many cancer-related deaths worldwide $(1,2)$. Although several treatments for GC including excision surgery, radiotherapy and chemotherapy have been developed recently, the clinical outcome continues to be poor in patients with advanced GC (1). The mortality of GC is intrinsically related to metastasis, in which angiogenesis plays

Correspondence to: Professor Wangjun Liao, Department of Oncology, Nanfang Hospital, Southern Medical University, 1838 North Guangzhou Avenue, Guangzhou, Guangdong 510515, P.R China

E-mail: nfyyliaowj@163.com

Key words: angiogenesis, gastric cancer, tribbles homolog 3, VEGF-A a crucial role (3). Angiogenesis is also indispensable to the continuous growth of the tumor. Therefore, one of the most promising yet challenging therapeutic approaches to cure GC is to develop safe, effective, and affordable anti-angiogenic therapies.

Tribbles homolog 3 (TRIB3, also named TRB3, NIPK, and SKIP3) belongs to the tribbles family of pseudokinases that were first identified in Drosophila to regulate cell division and migration (4-6). TRIB3 also participates in the activation of multiple signaling pathways, such as mitogen-activated protein kinase (MAPK) pathways (7-9). TRIB3 expression is upregulated by endoplasmic reticulum stress, hypoxia, and nutrient starvation $(6,10,11)$. Recent studies suggest that TRIB3 is a potential oncogene, as evidenced by its elevated expression in colorectal cancer (12), breast cancer (13), liver cancer and other cancer tissues (14). Furthermore, TRIB3 is associated with an adverse prognosis in these cancers. However, whether TRIB3 is involved in the development and progression of GC has not been reported.

Previous studies have shown that TRIB3 expression is closely related to the progression of type 2 diabetes mellitus, and that TRIB3 mediated apoptosis in islet $\beta$ cells, as well as insulin resistance. Together, these effects impair insulin-stimulated glucose uptake and maintain hyperglycemia in diabetes (15-17). Chronic hyperglycemia is a major initiator of diabetic angiopathy (18). Because impaired angiogenesis is a key pathological characteristic of diabetic microangiopathy (19), and it is well established that some inducers of TRIB3, such as endoplasmic reticulum stress, hypoxia, and glucose deprivation can also induce angiogenesis, we propose that the expression of TRIB3 is related to angiogenesis.

The present study analyzed TRIB3 expression in GC tissues from $191 \mathrm{GC}$ patients categorized from stage I to IV, to examine the role of TRIB3 in GC. The study also examined the relationship between TRIB3 and tumor angiogenesis. We found that TRIB3 expression was elevated in GC tissues, and that TRIB3 overexpression is correlated to the severity and poor prognosis in GC. We also showed that TRIB3 suppression downregulated the expression of VEGF-A in GC cells, which subsequently suppressed the recruitment of endothelial 
cells and formation of vessels. Thus, TRIB3 may be a promising target for anti-angiogenic therapy in GC.

\section{Materials and methods}

Patients and tumor tissue samples. Our study was approved by the Ethics Committee of Nanfang Hospital, the Southern Medical University. All tissues for diagnostic purposes were obtained with the consent of each patient diagnosed with primary GC and receiving resection surgery between 2004 and 2008. The clinical stages of the tumors were defined according to the National Comprehensive Cancer Network Guidelines (http://www.nccn.org/; version 1.2014, Gastric Cancer). No patient received any pre-operative chemotherapy or radiotherapy. A total of 191 tissue samples were used in this study.

Cells and cell culture. The human gastric epithelial cell line GES-1, and GC cell lines BGC803, BGC823, MGC803, MKN28, MKN45, and SGC7901 were obtained from Foleibao Biotechnology Development Company (Shanghai, China). Human umbilical vein endothelial cells (HUVECs) were purchased from Sciencell Research Laboratories (Carlsbad, CA, USA). All cells were cultured in RPMI-1640 medium (Hyclone, Logan, UT, USA) with $10 \%$ fetal bovine serum at $37^{\circ} \mathrm{C}$ and with $5 \% \mathrm{CO}_{2}$.

Immunohistochemistry. Immunohistochemical staining was used to evaluate the expression of TRIB3, VEGF-A, and CD31, as described previously (20). The primary antibodies used in this study were polyclonal rabbit antibodies for TRIB3 (Abcam, San Francisco, CA, USA), VEGF-A, and CD31 (ABclonal, Boston, MA, USA). The HRP-conjugated anti-rabbit secondary antibody was from CWBIO (Beijing, China). Specifically bound antibodies were detected with a 3,3'-diaminobenzidine staining kit (CWBIO). The percentage of positive cells was calculated and categorized as follows: 0 ( $0 \%$ of cells stained), 1 (1-25\%), 2 (26-50\%), 3 (51-75\%), or $4(76-100 \%)$. Staining intensity was visually scored as 0 (negative), 1 (weak), 2 (moderate), or 3 (strong). The final score (0-12) for the expression of TRIB3 and VEGF-A was calculated as the product of the percentage and the intensity scores for each case.

Tumor microvessel density (MVD) was determined by counting the number of endothelial cells positively stained for CD31, following the method described by Weidner et al (21). Slides were scanned initially at a low power (x100 magnification) in order to identify areas with higher vascular density (hot spots). Subsequently, counting of the stained microvessels was performed on four consecutive high power (x400 magnification) fields within the selected high density fields. Yellow-brown immunostained endothelial cells or an endothelial cell cluster that was clearly separated from adjacent microvessels were counted as vascular structures. The average number of microvessels counted in four $400 x$ fields provided the MVD value for each case.

Gene silencing with siRNAs. Three siRNA sequences against TRIB3 were purchased from RiboBio (Guangzhou, China). Transfection was carried out using Lipofectamine ${ }^{\circledR} 2000$ transfection reagent from Invitrogen (Carlsbad, CA, USA) following the manufacturer's recommended protocol. A negative control siRNA was used to examine the effect of transfection alone.

Western blotting. Proteins extracted from the tissues and cells were subjected to western blotting as described previously (20). Primary antibodies were polyclonal rabbit antibodies for TRIB3 (Abcam), VEGF-A (ABclonal), and $\alpha$-actin (Proteintech, Wuhan, China). A secondary fluorescent goat anti-rabbit antibody (LI-COR, Lincoln, NE, USA) was used in this study. The Odyssey imaging system (LI-COR) was used to scan the blots.

Quantitative real-time polymerase chain reaction ( $q R T-P C R$ ). Total RNA of the cultured cells was extracted using a TRIzol kit (Takara Bio, Inc., Shiga, Japan) according to the manufacturer's protocols. The First Strand cDNA synthesis kit (Takara Bio, Inc.) was used to synthesize cDNAs. qRT-PCR was performed using the SYBR-Green dye (Roche, Mannheim, Germany). The PCR primers used in this study were: 5'-ATTAGGCAGGGTCTGTCCTGTG-3' (TRIB3, sense), 5'-AGTATGGACCTGGGATTGTGGA-3' (antisense); 5'-CTT GCCTTGCTGCTCTACC-3' (VEGF-A, sense), 5'-CACACA GGATGGCTTGAAG-3' (antisense); 5'-TTCATTGACCTC AACTACATG-3' (GAPDH, sense), 5'-GTGGCAGTGATG GCATGGAC-3' (antisense).

Enzyme-linked immunosorbent assay (ELISA). GC cells (3x $10^{4} /$ well) were seeded in 24 -well plates and TRIB3 siRNA transfection was performed. The culture medium was collected $48 \mathrm{~h}$ after transfection and secretion of VEGF-A was determined using human VEGF-A ELISA kits (Baomanbio, Shanghai, China) according to the manufacturer's instructions. The results are presented as mean \pm standard deviation from three independent experiments.

HUVEC migration assay. GC cells ( $3 \times 10^{4} /$ well) were seeded in 24-well plates and TRIB3 siRNA transfection was performed. After culturing at $37^{\circ} \mathrm{C}$ for $48 \mathrm{~h}$, a chamber with a porous ( $8.0 \mathrm{~mm}$ pore size) polycarbonate membrane filter (Millipore Corp., Bedford, MA, USA) containing $4 \times 10^{4}$ HUVECs in $0.2 \mathrm{ml}$ RPMI-1640 medium without fetal bovine serum, was inserted into each well. HUVECs were fixed with $4 \%$ paraformaldehyde after co-culture for $12 \mathrm{~h}$ at $37^{\circ} \mathrm{C}$, and subsequently stained with $0.1 \%$ crystal violet for $30 \mathrm{~min}$. The results were observed under an inverted microscope.

Scratch wound healing assay. GC cells $\left(3 \times 10^{4} /\right.$ well) were seeded in 6-well plates and TRIB3 siRNA transfection was performed. The culture medium was collected $48 \mathrm{~h}$ after transfection. Trypsinized HUVECs were seeded in 6-well plates at a density of $3 \times 10^{5}$ cells per well. After reaching confluency, cultured cells were scratched with a sterile $200-\mu 1$ pipette tip. The cells were then cultured in the GC cell-conditioned medium. Wound closure was observed at 0,24 , and $48 \mathrm{~h}$ under an inverted microscope.

Tubule formation by HUVECs. HUVECs mixed with $100 \mu \mathrm{l}$ GC cell-conditioned medium were seeded at a density 
A
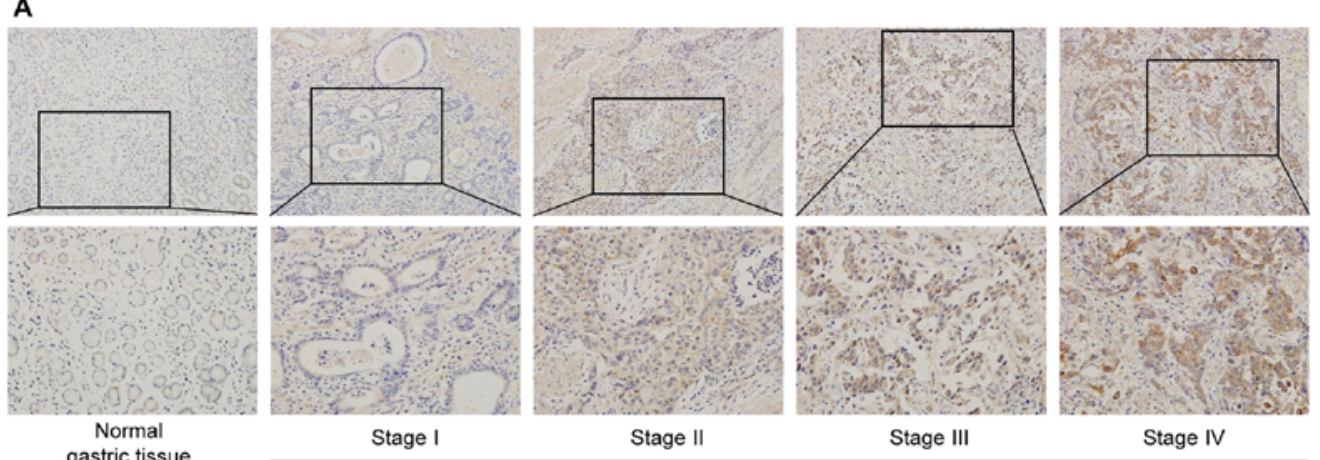

gastric tissue

Stage I

Stage II

Stage III

Stage IV

B

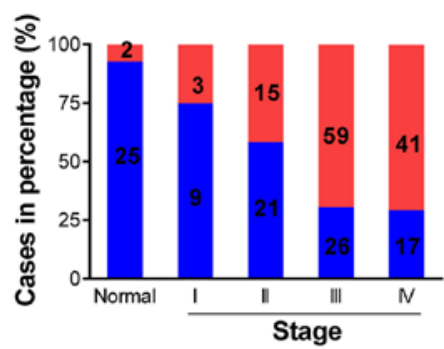

Gastric tumor tissues

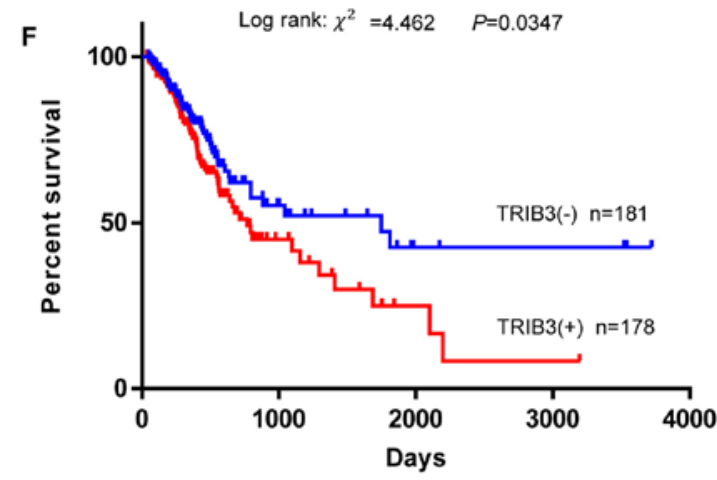

C

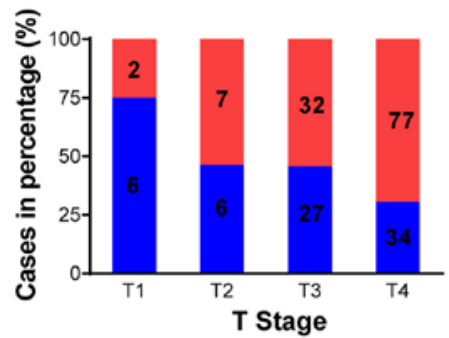

D

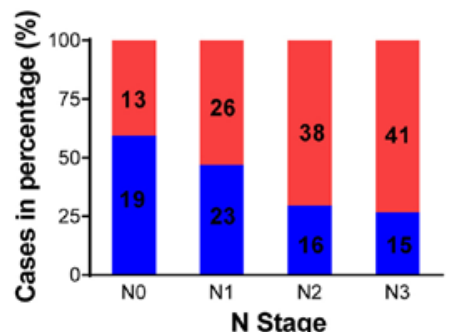

G

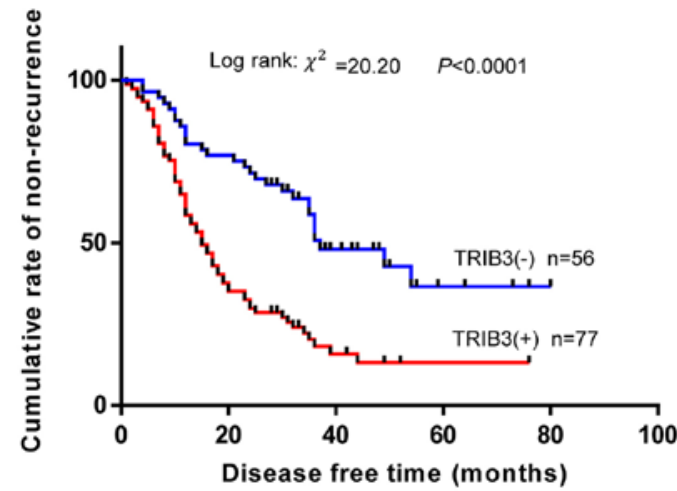

E

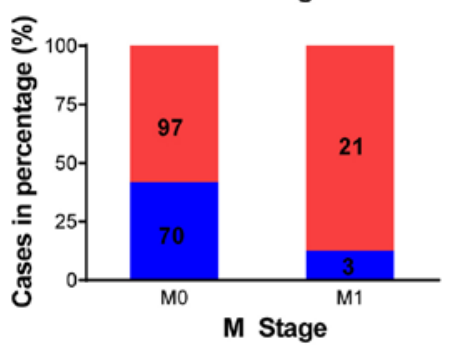

H

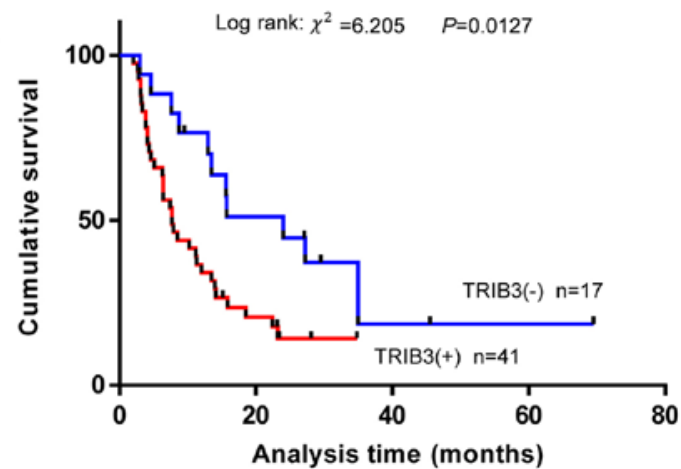

Figure 1. TRIB3 is elevated in advanced gastric cancer (GC) and is associated with a poor prognosis. (A) Representative examples of TRIB3 staining in normal gastric and GC tissues (magnification, x200 and x400, respectively). (B-E) The frequency of low and high expression levels of TRIB3 in GC categorized by (B) TNM stage, (C) T stage, (D) N stage, and (E) M stage. (F) Kaplan-Meier curves for overall survival duration of GC patients according to TRIB3 expression, using data from the TCGA database. (G) Kaplan-Meier curves for postoperative recurrence of cancer in patients with stage I-III GC according to the expression of TRIB3. (H) Overall survival of patients with stage IV GC according to TRIB3 expression.

of $2 \times 10^{4}$ cells per well in 96 -well plates containing $60 \mu 1$ solidified Matrigel (BD Biosciences, New York, NY, USA) and cultured for $6-8 \mathrm{~h}$ at $37^{\circ} \mathrm{C}$. Cultures were photographed under a microscope. The number of tubules was counted in three individual wells and presented as the mean \pm standard deviation. 
Statistical analyses. Statistical calculations were performed using SPSS 13.0 software (IBM, Chicago, IL, USA). Survival analysis was performed according to the Kaplan-Meier method. Differences of survival between groups were assessed with the log-rank test. The relationship between TRIB3 and MVD was evaluated by linear regression analysis. Student's t-test was performed to determine significant differences between two experimental groups. One-way ANOVA analysis was used to evaluate the statistical significance among multiple groups. $\mathrm{P}<0.05$ was considered to indicate a statistically significant result.

\section{Results}

TRIB3 expression is elevated in advanced GC and associated with a poor prognosis. In order to detect the expression of TRIB3, immunohistochemical staining was performed in 27 normal gastric tissues and 191 GC tissues. Patients were divided into two groups, low (score 0-5) and high (score $>5$ ) expression levels, according to the TRIB3 staining score. TRIB3 was markedly upregulated in GC compared with normal gastric tissues. High expression levels of TRIB3 were observed in $61.8 \%(118 / 191)$ of the GC tissues. This was significantly $>7.4 \%(2 / 27)$ seen in normal gastric tissues. Representative staining examples of normal gastric and GC tissues are shown in Fig. 1A.

The correlation analysis between pathological characteristics and expression levels of TRIB3 showed that high expression of TRIB3 was more frequent in patients with more advanced overall TNM stage $(\mathrm{P}=0.001$, Fig. $1 \mathrm{~B})$, $\mathrm{T}$ stage $(\mathrm{P}=0.029$, Fig. 1C), $\mathrm{N}$ stage $(\mathrm{P}=0.006$, Fig. 1D), and distant metastasis $(\mathrm{P}=0.006$, Fig. 1E). However, no statistical significance was found with age and gender $(\mathrm{P}=0.339$ and 0.578 , respectively, Table I).

Kaplan-Meier curves for overall survival duration of GC patients, according to TRIB3 expression levels using data from the TCGA database, showed that GC patients who had higher levels of TRIB3 expression exhibited a worse prognosis (log-rank $=4.462, \mathrm{P}=0.0347$, Fig. 1F). Similar results were observed in the present study where high expression levels of TRIB3 were significantly correlated with short time to recurrence among patients in stages I-III (log-rank $=20.20$, $\mathrm{P}<0.0001$, Fig. 1G), and short survival time among patients in stage IV (log-rank =6.205, P=0.0127, Fig. $1 \mathrm{H})$.

Overexpression of TRIB3 is associated with tumor angiogenesis in $G C$. To clarify the potential relationship between TRIB3 and angiogenesis, a total of $91 \mathrm{GC}$ tissues were immunostained for TRIB3 and angiogenesis-related markers. VEGF-A is an important hallmark of angiogenesis, while microvessels were identified by CD31 staining. Representative staining examples of low and high expression of TRIB3, VEGF-A, and CD31 in GC are shown in Fig. 2A. Tumors with high expression levels of TRIB3 had a significantly higher MVD than those with low expression levels (18.2 \pm 1.1 vs. 13.1 \pm 1.0 , $\mathrm{t}=3.261, \mathrm{P}=0.0016$, Fig. $2 \mathrm{~B})$. Similar results were observed in the groups where both TRIB3 and VEGF-A expression were elevated (22.4 \pm 1.4 vs. $12.3 \pm 0.82, \mathrm{t}=6.460, \mathrm{P}<0.0001$, Fig. $2 \mathrm{~B})$. Using data from the TCGA database, linear regression analysis revealed that TRIB3 and VEGF-A expressions were
Table I. Correlations between the clinicopathological parameters and expression of TRIB3.

\begin{tabular}{|c|c|c|c|c|c|}
\hline \multirow[b]{2}{*}{ Parameter } & \multirow[b]{2}{*}{$\mathrm{n}$} & \multicolumn{2}{|c|}{$\begin{array}{c}\text { Expression of } \\
\text { TRIB3 (n) }\end{array}$} & \multirow[b]{2}{*}{ P-value } & \multirow[b]{2}{*}{$\chi^{2}$} \\
\hline & & High & Low & & \\
\hline Gender & & & & 0.578 & 0.309 \\
\hline Male & 125 & 79 & 46 & & \\
\hline Female & 66 & 39 & 27 & & \\
\hline Age (years) & & & & 0.339 & 0.913 \\
\hline$\leq 55$ & 72 & 45 & 27 & & \\
\hline$>55$ & 119 & 66 & 53 & & \\
\hline TNM stage & & & & 0.001 & 17.089 \\
\hline I & 12 & 3 & 9 & & \\
\hline II & 36 & 15 & 21 & & \\
\hline III & 85 & 59 & 26 & & \\
\hline IV & 58 & 41 & 17 & & \\
\hline Tumor invasion & & & & 0.029 & 9.059 \\
\hline $\mathrm{T} 1$ & 8 & 2 & 6 & & \\
\hline $\mathrm{T} 2$ & 13 & 7 & 6 & & \\
\hline $\mathrm{T} 3$ & 59 & 32 & 27 & & \\
\hline $\mathrm{T} 4$ & 111 & 77 & 34 & & \\
\hline Lymph node & & & & & \\
\hline metastasis & & & & 0.006 & 12.431 \\
\hline NO & 32 & 13 & 19 & & \\
\hline N1 & 49 & 26 & 23 & & \\
\hline N2 & 54 & 38 & 16 & & \\
\hline N3 & 56 & 41 & 15 & & \\
\hline Distant metastasis & & & & 0.006 & 7.69 \\
\hline M0 & 167 & 97 & 70 & & \\
\hline M1 & 24 & 21 & 3 & & \\
\hline
\end{tabular}

significantly correlated ( $\mathrm{r}=0.416, \mathrm{P}<0.01$, Fig. $2 \mathrm{C})$. Similarly, a significant positive correlation between the expression of TRIB3 and VEGF-A was found in the present study $(r=0.468$, $\mathrm{P}<0.01$, Fig. 2D). TRIB3 positivity was also significantly correlated with MVD by linear regression analysis $(r=0.346$, $\mathrm{P}<0.01$, Fig. 2E).

Using Kaplan-Meier analysis with the log-rank test, we found that high expression of VEGF-A was significantly correlated with short time to recurrence among GC patients in stages I-III (log-rank =5.776, P=0.0162, Fig. 2F). Similar results were observed in patients expressing high levels of VEGF-A combined with high levels of TRIB3 (log-rank $=14.61$, $\mathrm{P}<0.0001$, Fig. 2G). In addition, high expression of VEGF-A was associated with short survival time among stage IV GC patients (log-rank $=10.77, \mathrm{P}=0.001$, Fig. $2 \mathrm{H}$ ). Similar results were observed in patients with high levels of both VEGF-A and TRIB3 (log-rank $=5.025, \mathrm{P}=0.025$, Fig. 2I).

Expression of TRIB3 and VEGF-A in GC surgical samples and $G C$ cell lines. To confirm the elevated expression of TRIB3 and VEGF-A in GC tissues, fresh surgically resected 
A
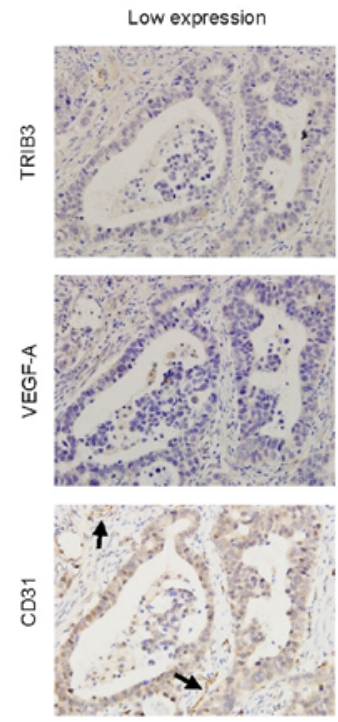

C

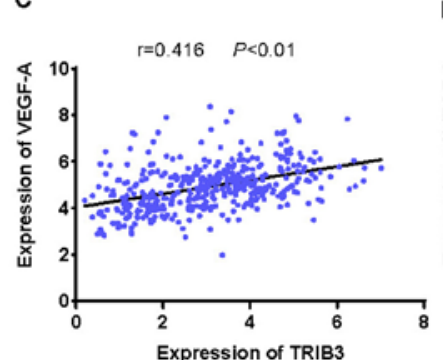

$\mathbf{F}$
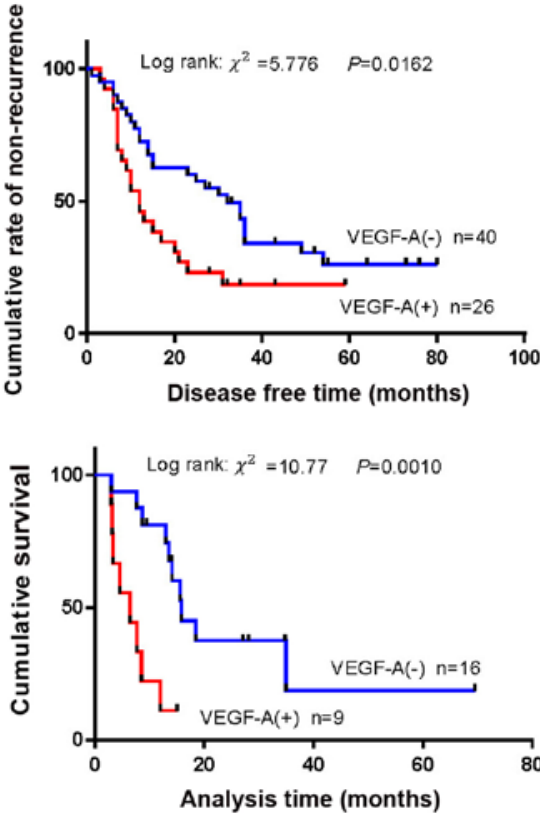

High expression
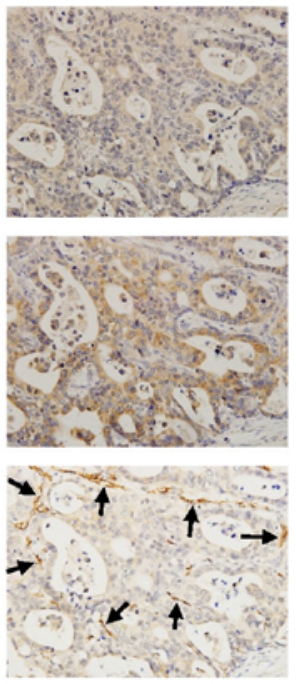

D

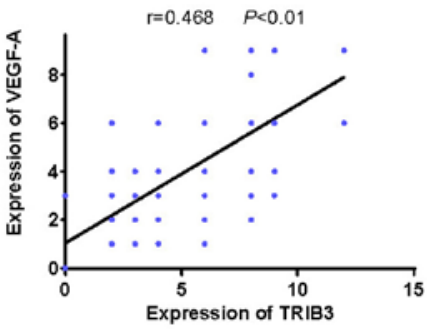

E

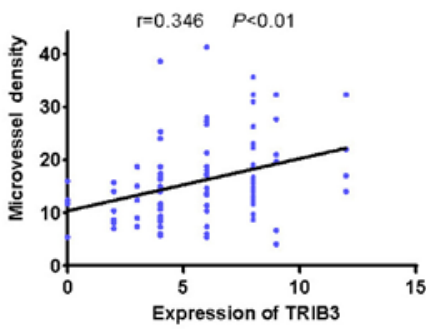

G
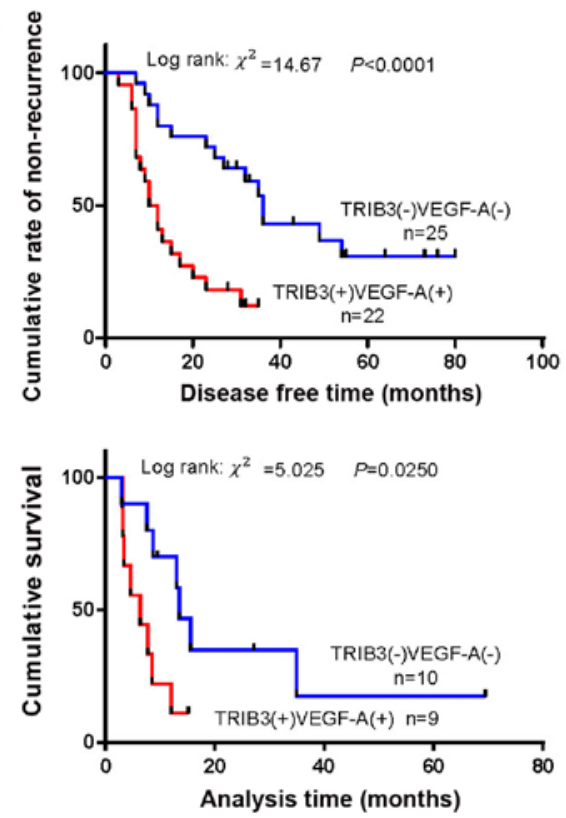

Figure 2. Expression of TRIB3 correlates with VEGF-A expression and microvessel density (MVD) in human gastric cancer (GC) tissues. (A) Representative staining examples of low and high expression levels of VEGF-A and MVD in GC with low or high TRIB3 expression. (B) MVD determined by CD31 staining in GC according to TRIB3 expression and TRIB3 combined with VEGF-A expression. Spearman's rank correlation analyses showing the correlation between the expression of TRIB3 and VEGF-A using (C) data from the TCGA database, and (D) data from immunohistochemical staining in this study. (E) Correlation between the expression of TRIB3 and MVD. Kaplan-Meier analyses for postoperative recurrence of patients with stage I-III GC according to the expression of (F) VEGF-A or (G) VEGF-A combined with TRIB3. Overall survival of patients with stage IV GC according to (H) VEGF-A expression and (I) VEGF-A combined with TRIB3. ${ }^{*} \mathrm{P}<0.05,{ }^{* *} \mathrm{P}<0.01$ compared with the control group.

specimens were analyzed by western blotting. Both TRIB3 and VEGF-A were expressed at higher levels in tumor tissues than in the adjacent non-tumorous gastric tissues (Fig. 3A). To better elucidate the expression profiles of TRIB3 and VEGF-A, we examined their mRNA and protein expression levels in a human gastric epithelial cell line (GES-1) and six GC 

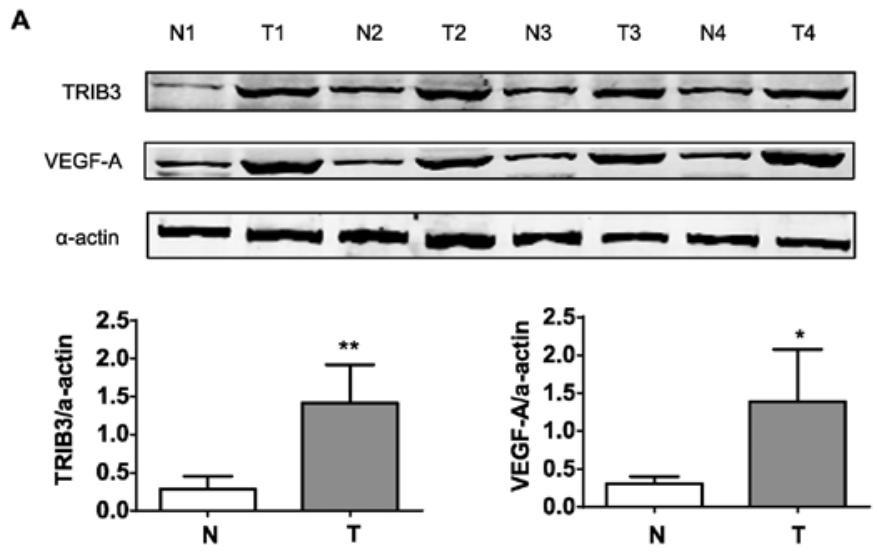

B
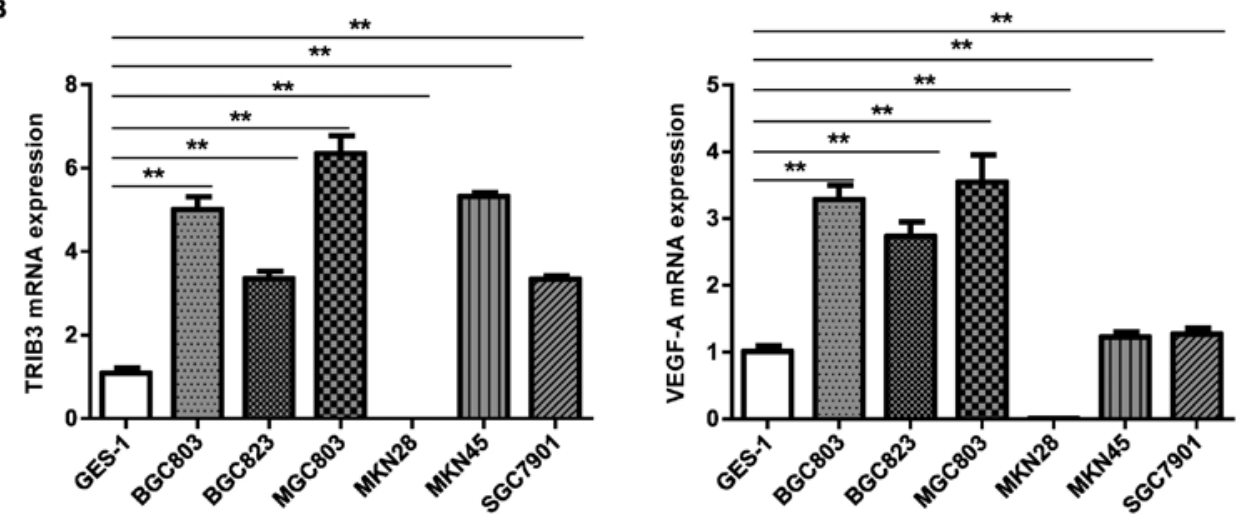

c

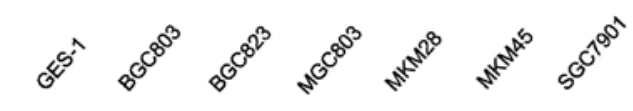

TRIB3

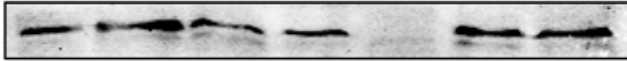

VEGF-A

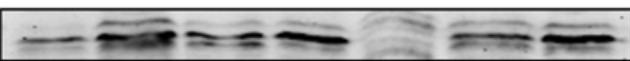

a-actin
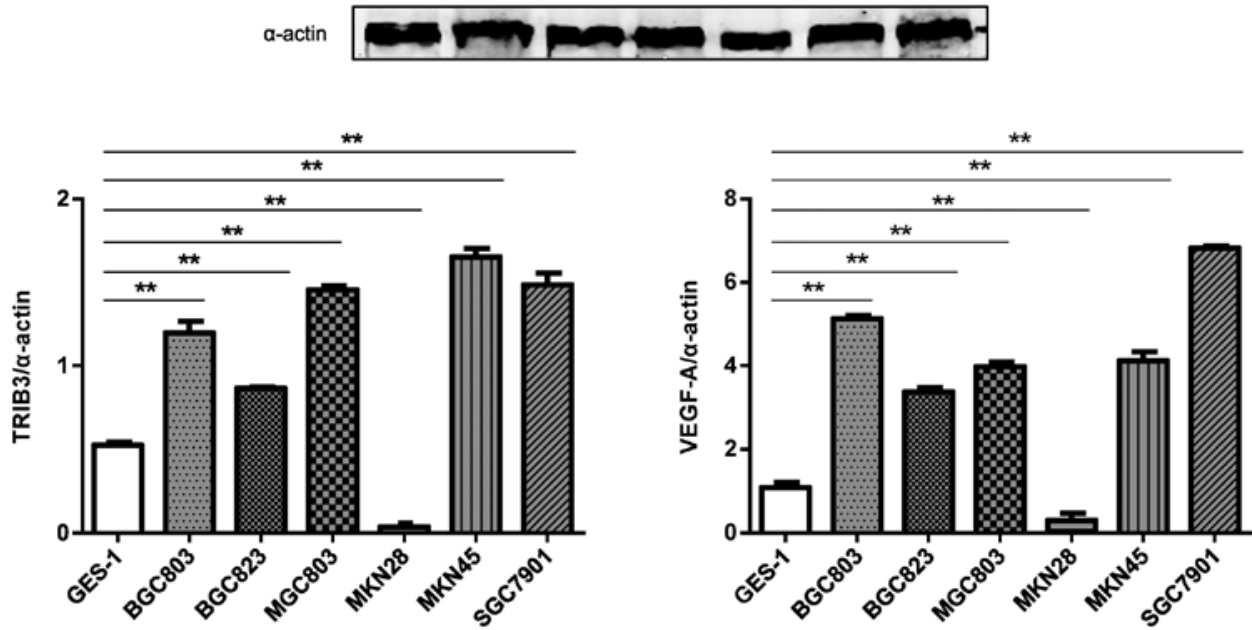

Figure 3. Expression of TRIB3 and VEGF-A in gastric cancer (GC) surgical samples and GC cell lines. (A) Western blotting of TRIB3 and VEGF-A in GC tissues (T) and adjacent non-tumor tissues (N). Expression levels of TRIB3 and VEGF-A in the human gastric epithelial cell line GES-1 and six GC cell lines were determined by (B) qRT-PCR and (C) western blot analyses. ${ }^{*} \mathrm{P}<0.05,{ }^{* * *} \mathrm{P}<0.01$ compared with the control group.

cell lines (BGC803, BGC823, MGC803, MKN28, MKN45, and SGC7901). Except for MKN28 cells, the expression of TRIB3 mRNA was higher in GC (3- to 7-fold) than in GES-1 cells (Fig. 3B). Interestingly, a similar trend was observed in the expression of VEGF-A mRNA (Fig. 3B). The expression of
TRIB3 and VEGF-A proteins were consistent with the mRNA results (Fig. 3C).

TRIB3 silencing downregulates the expression of VEGF-A in GC cells. The ability of three different TRIB3-siRNA 
A

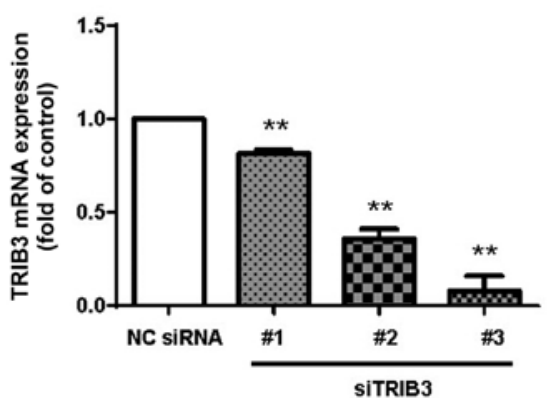

B

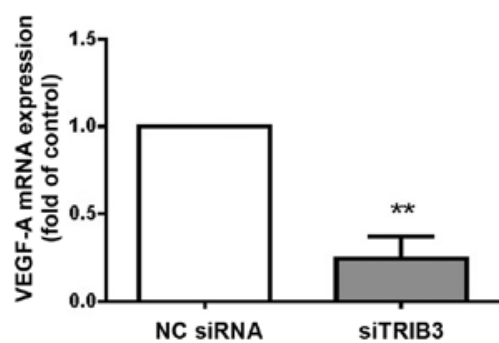

MKN45

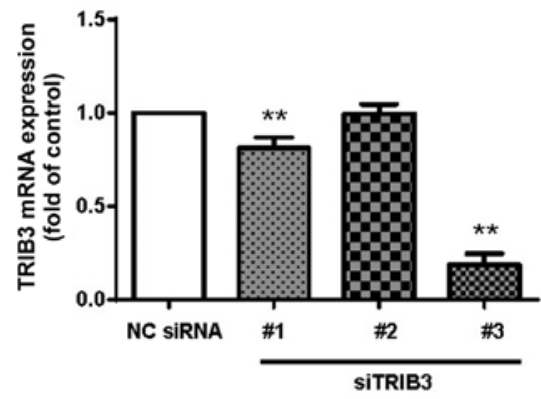

MKN45

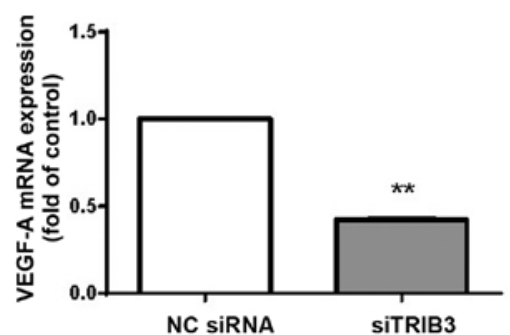

C

BGC823
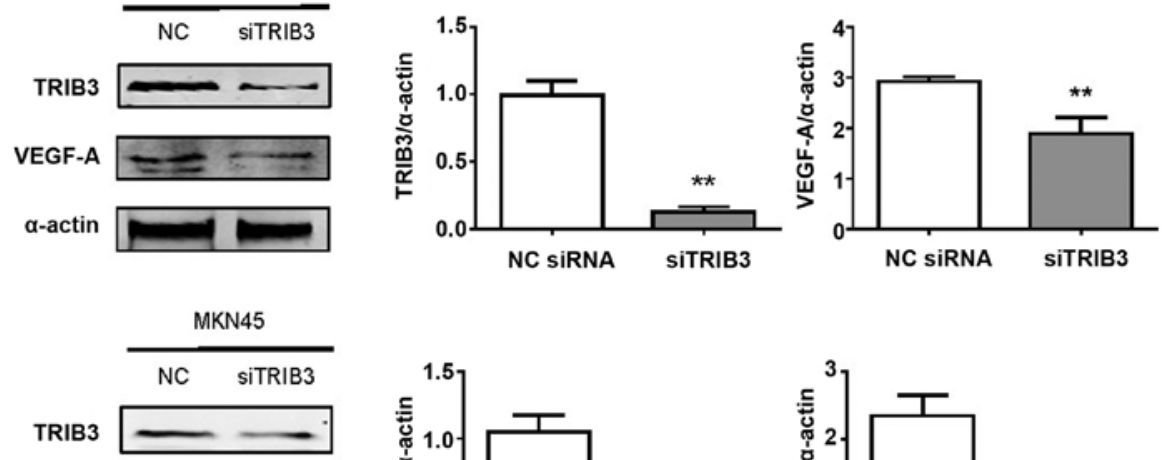

VEGF-A -1SO

$\alpha$-actin
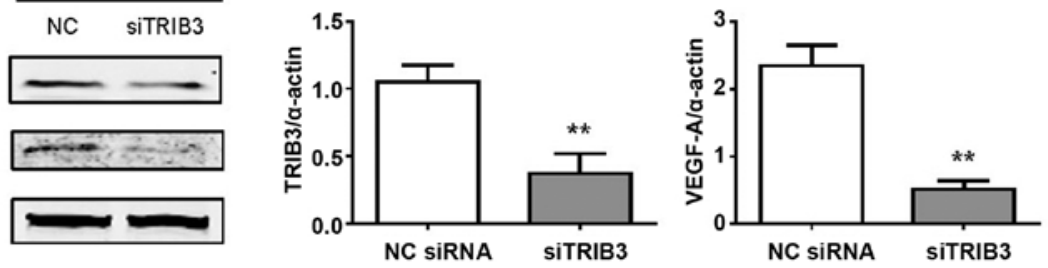

D BGC823

MKN45
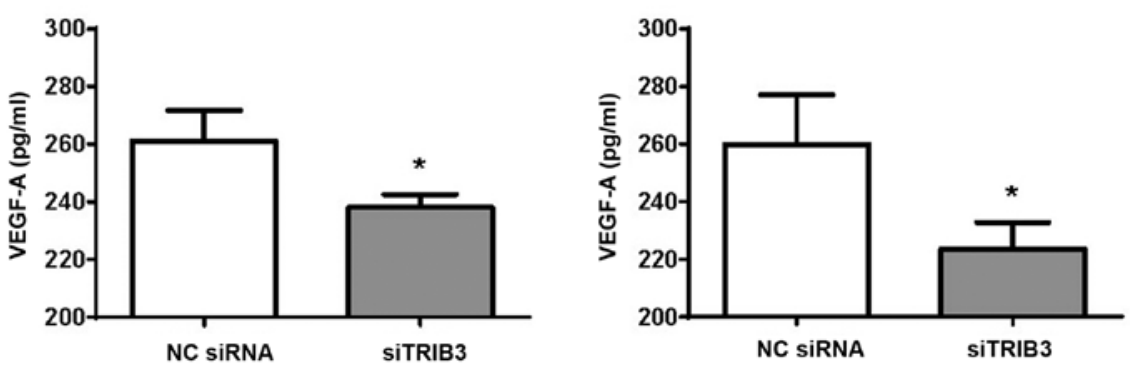

Figure 4. TRIB3 silencing downregulates the expression of VEGF-A in gastric cancer cell lines. (A) The ability of three different siRNA sequences against TRIB3 to downregulate this gene in the BGC823 and MKN45 cells was determined by qRT-PCR. (B) VEGF-A mRNA was downregulated in the BGC823 and MKN45 cells with TRIB3-silencing siRNAs. (C) VEGF-A protein expression was downregulated by TRIB3-silencing siRNAs in the BGC823 and MKN45 cells. (D) ELISA analyses of VEGF-A secreted protein levels using conditioned media derived from GC cells transfected with the negative control (NC) or TRIB3 siRNA. ${ }^{*} \mathrm{P}<0.05,{ }^{* *} \mathrm{P}<0.01$ compared with the control group.

sequences to downregulate TRIB3 was determined by qRT-PCR. Only siTRIB3-\#3 consistently suppressed the expression of TRIB3 mRNA in the BGC823 and MKN45 cells (Fig. 4A). Interestingly, the expression of VEGF-A mRNA was also downregulated by TRIB3-\#3-siRNA in the BGC823 and MKN45 cells $(\mathrm{P}=0.0005$ and $\mathrm{P}<0.0001$, respectively, Fig. 4B). Both TRIB3 and VEGF-A protein levels were significantly reduced by TRIB3-\#3-siRNA in the BGC823 ( $\mathrm{P}=0.0002$ and 0.0062 , respectively, Fig. 4C) and MKN45 ( $\mathrm{P}=0.0036$ and 0.0006 , respectively, Fig. 4C) 
A

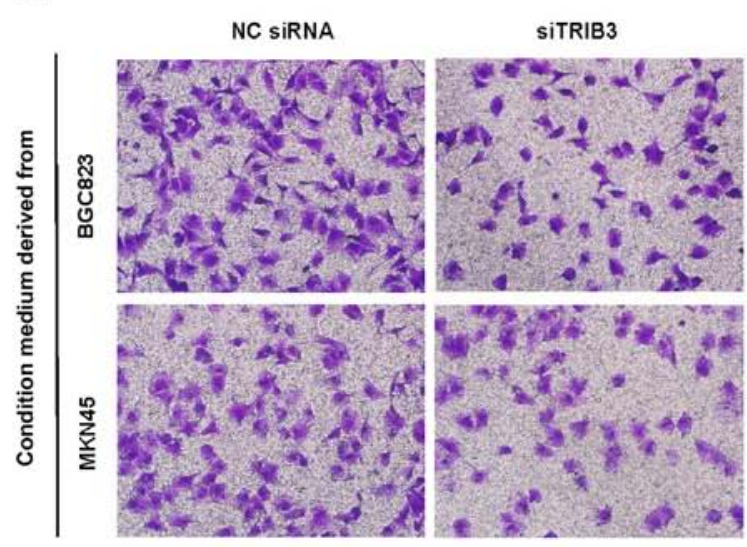

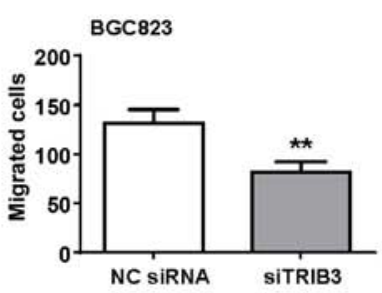

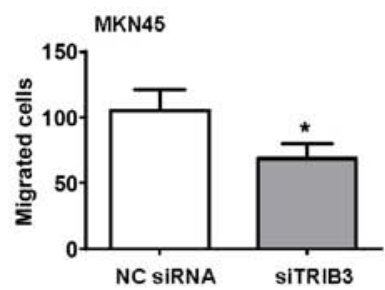

B Condition medium derived from

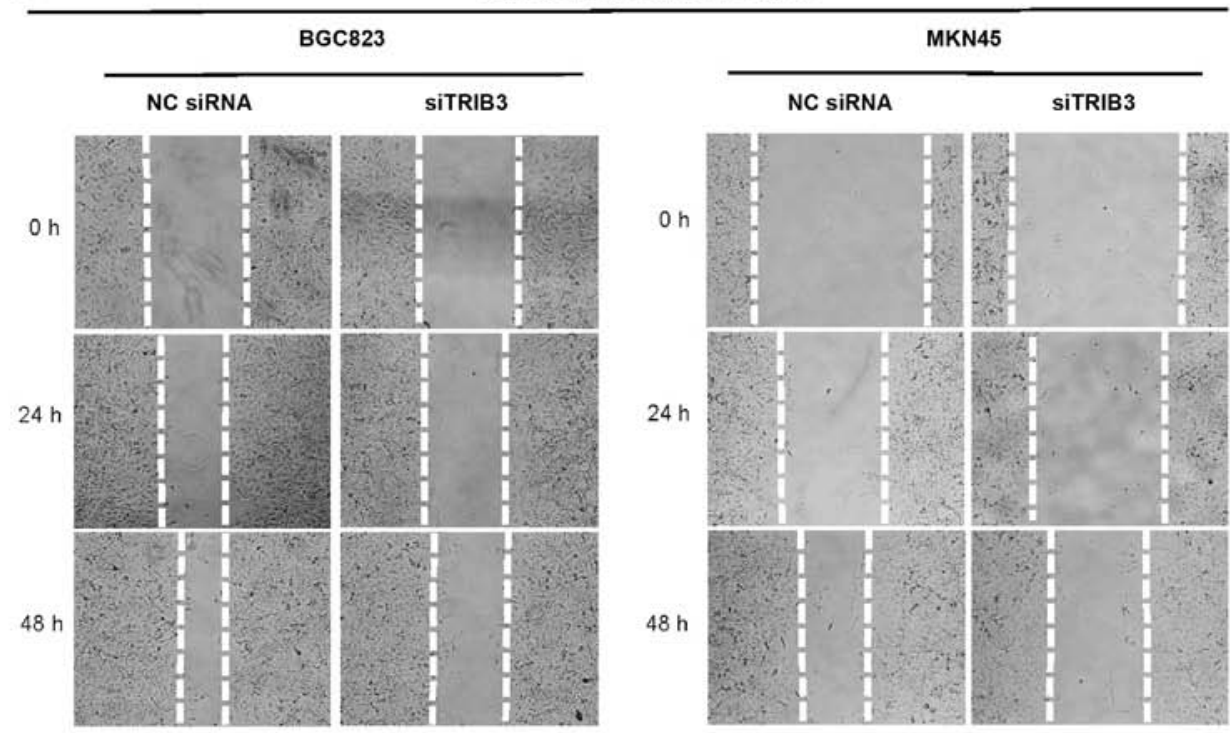

C
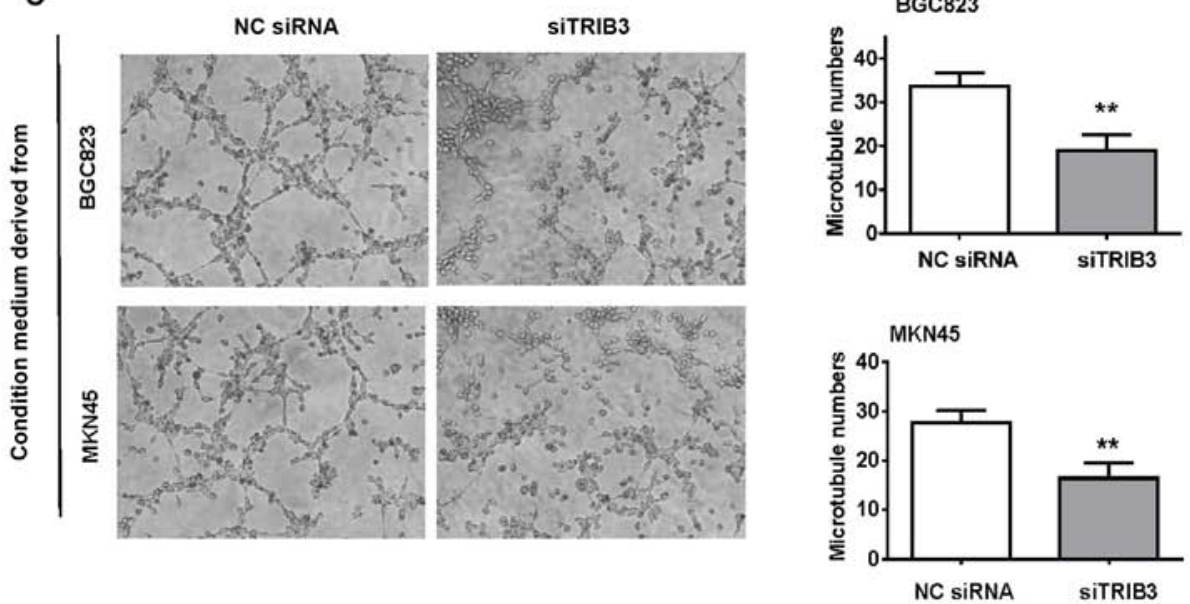

Figure 5. TRIB3 silencing downregulates angiogenesis in vitro. (A) Transwell migration assay with HUVECs in the upper chamber and GC cell-conditioned medium in the lower chamber. (B) Scratch wound healing assay in HUVECS using a linear scrape with a pipette tip followed by addition of GC cell-conditioned medium for $48 \mathrm{~h}$. (C) HUVECs tube formation assay. ${ }^{*} \mathrm{P}<0.05,{ }^{* *} \mathrm{P}<0.01$ compared with the control group.

cells. ELISA analyses showed that secreted VEGF-A protein was also decreased in the TRIB3-knockdown GC cells, when compared with control cells $(\mathrm{P}=0.0279$ and 0.0329 in BGC823 and MKN45 cells, respectively, Fig. 4D). These data showed that TRIB3 silencing contributed to the downregula- tion of VEGF-A mRNA and protein expression in the GC cells.

TRIB3 silencing in GC cells compromises their ability to recruit endothelial cells via soluble factors. Angiogenesis 
is dependent on the proliferation and migration of vascular endothelial cells. To examine whether TRIB3 silencing influenced angiogenesis, we evaluated the responses of HUVECs in terms of migration and tubule formation. The migration capacity of HUVECs was altered after co-culturing with GC cell-conditioned media as shown by both the migration and scratch wound healing assays (Fig. 5A and B). Silencing of TRIB3 in the GC cells significantly impaired their ability to recruit HUVECs. Significant differences in the tubule-like structure formation capacity of HUVECs were also observed between the control siRNA and TRIB3-\#3-siRNA groups (Fig. 5C).

\section{Discussion}

The purpose of the present study was to investigate the oncological significance of TRIB3 in GC. We evaluated the expression of TRIB3 in patients with GC in order to determine correlations with pathological variables, including TNM stage, survival time of patients, and incidence of cancer recurrence, and to examine the relationship with angiogenesis.

Tribbles, first identified in Drosophila, are members of the pseudokinase family of proteins with no associated kinase activity. Instead of direct phosphorylation of target proteins, tribbles act as adaptor or scaffold proteins, as well as decoy kinases, that impede the function of other kinases through obstructive binding $(22,23)$. Currently, three mammalian homologs of the tribbles gene are known. One of these, TRIB3, plays an important role in multiple signaling pathways, coordinating crucial cellular processes such as apoptosis, glucose and lipid metabolism, adipocyte differentiation, and cell stress (15,24-27). TRIB3 is overexpressed in many cancers, but whether TRIB3 is upregulated in GC has not been reported previously.

To the best of our knowledge, this is the first study to evaluate the expression of TRIB3 in GC patients and GC cell lines, and examine the role of TRIB3 in tumor angiogenesis. Our results showed that TRIB3 was significantly upregulated in GC tissues compared with its level in adjacent non-tumor tissues, and that high expression levels of TRIB3 were more frequent in patients at a more advanced overall TNM stage, $\mathrm{T}$ stage, $\mathrm{N}$ stage, and with distant metastasis. High expression levels of TRIB3 predicted high cancer recurrence and mortality for GC patients. At the cellular level, TRIB3 was constitutively expressed at higher levels in all GC cell lines tested, except MKN28, when compared to a gastric epithelial cell line. These data show the potential utility of TRIB3 as a prognostic marker for GC.

In colorectal cancer, TRIB3 is a prognostic marker and has functional relevance to cell growth (12). In breast cancer, TRIB3 is involved in the ability of cancer cells to survive in hypoxic conditions and is associated with a poor prognosis (13). Similar relationships were noted in other types of cancer $(14,28,29)$. Knockdown of TRIB3 in tumor cells significantly inhibited the invasive and metastatic ability of the cells by promoting mesenchymal-epithelial transition (30). The finding of elevated TRIB3 expression in different malignant tumors supports the clinical results of our study, implying that TRIB3 may be overexpressed ubiquitously in tumors and play an important role in promoting tumor progression.
The role of TRIB3 in multiple signaling pathways, such as the MAPK pathway, combined with its role in hypoxia, metabolism, and cell stress processes, originally spurred us to investigate its role in angiogenesis. Because angiogenesis is dependent on the proliferation and migration of vascular endothelial cells, where VEGF-A plays an extremely important role, specific studies were conducted to identify the role of TRIB3 in angiogenesis. Firstly, immunohistochemical staining of $91 \mathrm{GC}$ tissues showed a significant positive correlation between the expression of TRIB3 and VEGF-A. In addition, tumors with high expression levels of TRIB3 had a higher MVD than those with low expression levels of TRIB3. At the cellular level, the expression of TRIB3 and VEGF-A were, with only one exception, consistent in a human gastric epithelial cell line and GC cell lines, suggesting that there is a correlation between the expression levels of the two genes. Both mRNA and protein expression levels of VEGF-A were significantly reduced by TRIB3-siRNA in GC cells. These data strongly suggested that TRIB3 silencing downregulated the expression of VEGF-A in GC cells. Further studies showed that TRIB3 silencing in GC cells significantly impaired their ability to recruit endothelial cells via soluble factors, implying that TRIB3 silencing may downregulate angiogenesis in vitro.

Overall, tumor angiogenesis is a complex process. Whether TRIB3 directly or indirectly modulates VEGF-A is still unclear and requires further investigation.

In summary, our study shows that: i) the expression of TRIB3 is significantly upregulated in GC; ii) high expression levels of TRIB3 are associated with a shorter survival time and a higher incidence of cancer recurrence and metastasis; and iii) TRIB3 silencing contributes to the downregulation of VEGF-A expression and angiogenesis in GC. Taken together, our data suggest that suppression of TRIB3 may be a novel anti-angiogenic approach for GC treatment.

\section{Acknowledgements}

This study was supported by grants from the National Natural Science Foundation of China (nos. 31271564 and 81472314), the Special Foundation for National Clinical Specialties of China (to The Department of Oncology, Nanfang Hospital), and the Team Program of Natural Science Foundation of Guangdong Province, China (no. 2011030003134).

\section{References}

1. Bang YJ, Van Cutsem E, Feyereislova A, Chung HC, Shen L, Sawaki A, Lordick F, Ohtsu A, Omuro Y, Satoh T, et al: ToGA Trial Investigators: Trastuzumab in combination with chemotherapy versus chemotherapy alone for treatment of HER2-positive advanced gastric or gastro-oesophageal junction cancer (ToGA): A phase 3, open-label, randomised controlled trial. Lancet 376: 687-697, 2010.

2. Shen L, Shan YS, Hu HM, Price TJ, Sirohi B, Yeh KH, Yang YH, Sano T, Yang HK, Zhang X, et al: Management of gastric cancer in Asia: Resource-stratified guidelines. Lancet Oncol 14: e535-e547, 2013.

3. Gacche RN: Compensatory angiogenesis and tumor refractoriness. Oncogenesis 4: e153, 2015.

4. Grosshans J and Wieschaus E: A genetic link between morphogenesis and cell division during formation of the ventral furrow in Drosophila. Cell 101: 523-531, 2000.

5. Mata J, Curado S, Ephrussi A and Rørth P: Tribbles coordinates mitosis and morphogenesis in Drosophila by regulating string/CDC25 proteolysis. Cell 101: 511-522, 2000. 
6. Bowers AJ, Scully S and Boylan JF: SKIP3, a novel Drosophila tribbles ortholog, is overexpressed in human tumors and is regulated by hypoxia. Oncogene 22: 2823-2835, 2003.

7. Kiss-Toth E, Bagstaff SM, Sung HY, Jozsa V, Dempsey C, Caunt JC, Oxley KM, Wyllie DH, Polgar T, Harte M, et al: Human tribbles, a protein family controlling mitogen-activated protein kinase cascades. J Biol Chem 279: 42703-42708, 2004.

8. Rzymski T, Paantjens A, Bod J and Harris AL: Multiple pathways are involved in the anoxia response of SKIP3 including HuR-regulated RNA stability, NF-kappaB and ATF4. Oncogene 27: 4532-4543, 2008

9. Du K, Herzig S, Kulkarni RN and Montminy M: TRB3: A tribbles homolog that inhibits Akt/PKB activation by insulin in liver. Science 300: 1574-1577, 2003.

10. Ord D and Ord T: Characterization of human NIPK (TRB3, SKIP3) gene activation in stressful conditions. Biochem Biophys Res Commun 330: 210-218, 2005.

11. Schwarzer R, Dames S, Tondera D, Klippel A and Kaufmann J: TRB3 is a PI 3-kinase dependent indicator for nutrient starvation. Cell Signal 18: 899-909, 2006.

12. Miyoshi N, Ishii H, Mimori K, Takatsuno Y, Kim H, Hirose H, Sekimoto M, Doki Y and Mori M: Abnormal expression of TRIB3 in colorectal cancer: A novel marker for prognosis. Br J Cancer 101: 1664-1670, 2009.

13. Wennemers M, Bussink J, Scheijen B, Nagtegaal ID, van Laarhoven HW, Raleigh JA, Varia MA, Heuvel JJ, Rouschop KM, Sweep FC, et al: Tribbles homolog 3 denotes a poor prognosis in breast cancer and is involved in hypoxia response. Breast Cancer Res 13: R82, 2011.

14. Hua F,LiK, Yu JJ,Lv XX, Yan J,Zhang XW, Sun W,Lin H, Shang S, Wang F, et al: TRB3 links insulin/IGF to tumour promotion by interacting with p62 and impeding autophagic/proteasomal degradations. Nat Commun 6: 7951, 2015.

15. Fang N, Zhang W, Xu S, Lin H, Wang Z, Liu H, Fang Q, Li C, Peng L and Lou J: TRIB3 alters endoplasmic reticulum stress-induced $\beta$-cell apoptosis via the $\mathrm{NF}-\kappa \mathrm{B}$ pathway. Metabolism 63: 822-830, 2014.

16. Zhang W, Liu J, Tian L, Liu Q, Fu Y and Garvey WT: TRIB3 mediates glucose-induced insulin resistance via a mechanism that requires the hexosamine biosynthetic pathway. Diabetes 62 : 4192-4200, 2013.

17. Liu J, Zhang W, Chuang GC, Hill HS, Tian L, Fu Y, Moellering DR and Garvey WT: Role of TRIB3 in regulation of insulin sensitivity and nutrient metabolism during short-term fasting and nutrient excess. Am J Physiol Endocrinol Metab 303: E908-E916, 2012.
18. Yamagishi S and Imaizumi T: Diabetic vascular complications: Pathophysiology, biochemical basis and potential therapeutic strategy. Curr Pharm Des 11: 2279-2299, 2005.

19. Cheng R and Ma JX: Angiogenesis in diabetes and obesity. Rev Endocr Metab Disord 16: 67-75, 2015

20. Wang L, Wu Y, Lin L, Liu P, Huang H, Liao W, Zheng D, Zuo Q, Sun L, Huang N, et al: Metastasis-associated in colon cancer-1 upregulation predicts a poor prognosis of gastric cancer, and promotes tumor cell proliferation and invasion. Int J Cancer 133: 1419-1430, 2013

21. Weidner N, Semple JP, Welch WR and Folkman J: Tumor angiogenesis and metastasis-correlation in invasive breast carcinoma. N Engl J Med 324: 1-8, 1991.

22. Yokoyama T and Nakamura T: Tribbles in disease: Signaling pathways important for cellular function and neoplastic transformation. Cancer Sci 102: 1115-1122, 2011

23. Lohan $\mathrm{F}$ and Keeshan $\mathrm{K}$ : The functionally diverse roles of tribbles. Biochem Soc Trans 41: 1096-1100, 2013

24. Örd T, Örd D, Adler P, Vilo J and Örd T: TRIB3 enhances cell viability during glucose deprivation in HEK293-derived cells by upregulating IGFBP2, a novel nutrient deficiency survival factor. Biochim Biophys Acta 1853: 2492-2505, 2015.

25. Bezy O, Vernochet C, Gesta S, Farmer SR and Kahn CR: TRB3 blocks adipocyte differentiation through the inhibition of C/EBPbeta transcriptional activity. Mol Cell Biol 27: 6818-6831, 2007.

26. Guo L, Guo ZX, Gong HP, Shang YY, Zhong M, Zhang Y and Zhang W: Tribbles homolog 3 is induced by high glucose and associated with apoptosis in human endothelial cells. Mol Med Rep 12: 1963-1970, 2015.

27. Brisard D, Chesnel F, Elis S, Desmarchais A, Sánchez-Lazo L, Chasles M, Maillard V and Uzbekova S: Tribbles expression in cumulus cells is related to oocyte maturation and fatty acid metabolism. J Ovarian Res 7: 44, 2014.

28. Zhang J, Wen HJ, Guo ZM, Zeng MS, Li MZ, Jiang YE, He XG and Sun CZ: TRB3 overexpression due to endoplasmic reticulum stress inhibits AKT kinase activation of tongue squamous cell carcinoma. Oral Oncol 47: 934-939, 2011.

29. Zhou H, Luo Y, Chen JH, Hu J, Luo YZ, Wang W, Zeng Y and Xiao L: Knockdown of TRB3 induces apoptosis in human lung adenocarcinoma cells through regulation of Notch 1 expression. Mol Med Rep 8: 47-52, 2013.

30. Hua F, Mu R, Liu J, Xue J, Wang Z, Lin H, Yang H, Chen X and $\mathrm{Hu} \mathrm{Z}$ : TRB3 interacts with SMAD3 promoting tumor cell migration and invasion. J Cell Sci 124: 3235-3246, 2011. 